\title{
THE DEVELOPMENT OF FLUORESCENCE MICROSCOPY FOR TUBERCLE BACILLI AND ITS USE AS AN ADJUNCT TO HISTOLOGICAL ROUTINE
}

\author{
BY \\ D. M. MCCLURE \\ From the Clinical Laboratories, the Victoria Infirmary, Glasgow
}

(RECEIVED FOR PUBLICATION FEBRUARY 4, 1953)

The microscopic demonstration of the tubercle baciilus by the Ziehl-Neelsen technique has always entailed heavy demands on the time and concentration of the observer, but in recent years fluorescence microscopy has offered a means of greatly facilitating this task. The spontaneous fluorescence of $\boldsymbol{M}$. tuberculosis under ultra-violet microscopy was first observed by Kaiserling (1917), but the use of fluorochrome staining is much more recent (Hagemann, 1937), and it is with this latter technique that this paper is concerned. The essential requirements are a high intensity light source and an appropriate system of filters. A blue filter eliminates the long wavelengths. The short wavelength rays elicit fluorescence from the stained preparation, and an orange eyepiece filter then allows only the fluorescence to reach the observer. The bacilli are seen as bright rods against a dark background, and are easily seen with low magnifications, permitting a much more rapid survey than with oil immersion objectives. For staining, Hagemann first employed berberine sulphate, but later he reported superior results with auramine O (Hagemann, 1938), and this has become the standard fluorochrome for $M$. tuberculosis.

In reviewing the literature it is proposed to consider (a) the apparatus used, (b) the staining methods, and $(c)$ the practical applications and results, and to comment on these before detailing the author's investigations.

\section{Apparatus}

The equipment used by Hagemann, incorporating an arc lamp and quartz lenses, was not generally available, but in 1941 a number of authors reported that auramine gave adequate fluorescence with a low-voltage/high-amperage filament lamp or a projection lamp, and a normal optical system (Bogen, 1941 ; Lind and Shaugh- nessy, 1941-2 ; Richards and Miller, 1941 ; Richards, Kline, and Leach, 1941). With this simplified equipment the technique was more widely adopted. Graham (1942) recommended a 500 w. projection lamp, though others preferred the mercury vapour lamp (Kloeck and Sweany, 1943 ; Stevens, 1945). Of the latter type, however, the $125 \mathrm{w}$. commercial " black lamp" did not give full field illumination with low-power objectives (Lempert, 1944; Norman and Jelks, 1945). Spectrographic investigations by Graham (1943) and Hughes (1943) showed that the effective wavelengths for the fluorescence of auramine extend well above the ultra-violet, and that the mercury vapour lamp is not an essential.

Concerning filters, Graham rejected blue glass types, and used a solution of copper sulphate and ammonia. This has been accepted generally, and, while a container with optically flat glass is desirable, an ordinary medicine bottle can be used (Andrew, Wilson, and Stuart, 1947 ; Matthaei, 1950). For the orange filter, glass and gelatin types have been equally effective.

References to condensers are few. Matthaei (1950) advises an "abbe" type to avoid any fluorescence from the cement between multiple lenses, which also absorb more light, and he specifies glycerol immersion of the condenser for maximum light transmission. Crossmon and Loewenstein (1943) recommend a dark-ground stop, but are alone in doing so.

The objectives employed in fluorescence work are those in normal use. For the $4 \mathrm{~mm}$. objective, one of N.A. 0.85 is preferable to N.A. 0.7 (Richards et al., 1941 ; Hughes, 1943). Opinions differ concerning fluorite and apochromatic lenses. Graham (1943) and Matthaei (1950) find them unsatisfactory, but Metcalf and Patton (1944) obtained good results, as did Hagemann in his original work. The use of an immersion objective 
has been mentioned (Kolmer, Spaulding, and Robinson, 1951), but in general there is no need for any power higher than $4 \mathrm{~mm}$.

The recommendations as to eyepieces differ widely. These fall into two groups: eyepieces of the powers in everyday use, and those of very much higher powers. Hagemann used a range from $3 \times$ to $10 \times$, and Bogen (1941) one of $5 \times$. At the other extreme, the use of eyepieces of $20 \times$ is recorded by Richards et al. (1941), Lind and Shaughnessy (1941-2), Hughes (1943), Lind (1949), and Popper and Szanto (1950). Lind even refers to one of $30 \times$. This subject will be discussed later with reference to my own experience.

Matthaei (1950) has observed that binocular microscopes differ widely in their capacity to transmit light in the blue-violet range. This may be attributable to the reflecting prisms, as no comparable variations have been recorded between the objectives and eyepieces of different manufacturers. In any case, a monocular instrument is to be preferred.

\section{Staining Methods}

With Hagemann's stain of 1 in 1,000 auramine in $5 \%$ phenol, the staining time was 15 minutes, followed by decolorizing in acid alcohol; the specimen was examined dry. The earlier modifications of this method used higher concentrations of auramine and heat while staining (Crossmon and Loewenstein, 1943). Background fluorescence in smears was found to be troublesome, and doubly so with tissue sections (Finke, 1940). Herrmann (1938) introduced counterstaining with potassium permanganate and Löffler's methylene blue. This found immediate favour, and has been specified as recently as 1950 by Popper and Szanto.

The first double fluorochrome method was that of Schallock (1940), who counterstained with thiazol yellow. Hughes (1946) used a combination of auramine $\mathrm{O}$, rhodamine $\mathrm{B}$, and acridine yellow, which showed bright golden yellow organisms on a green background. Graham (1943) referred to rhodamine, but did not adopt it in practice. Hughes emphasized that acridine yellow was unreliable, and so the logical step of omitting it was taken by Matthaei (1950), who also increased the concentration of auramine and rhodamine. $\mathrm{He}$ reported excellent results.

The acid alcohol solutions have also been subject to individual preference. Lee (1944), after trials, chose one of $25 \%$ sulphuric acid in $70 \%$ ethanol. Most workers, however, have found this

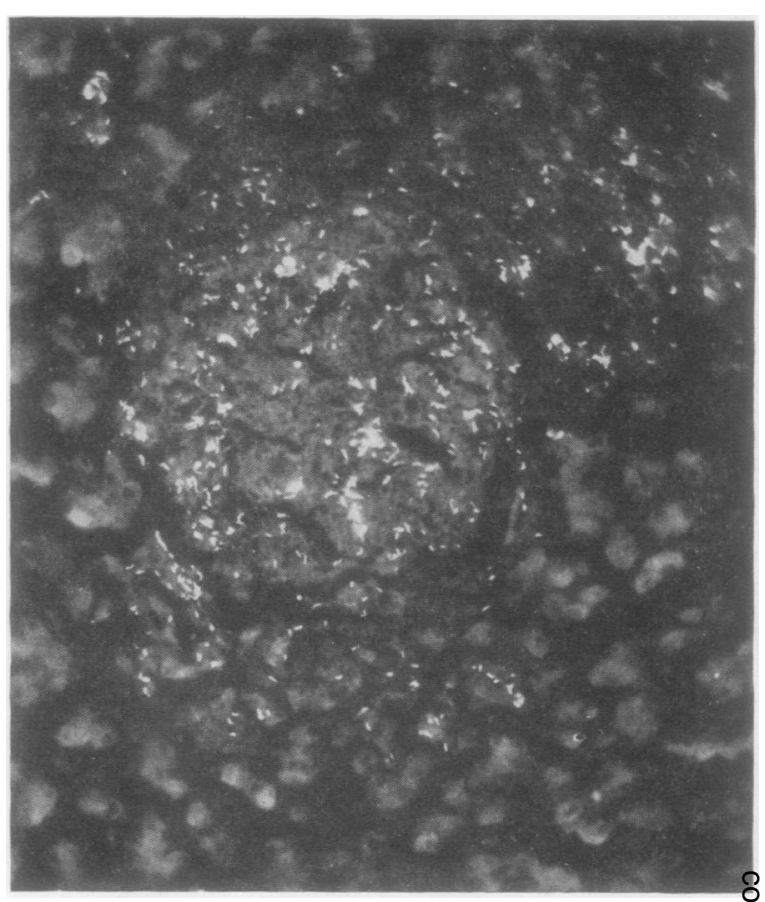

FIG. 1.-Miliary tuberculous focus in liver. Auramine-rhodamine stain, 360 .

too rapid and difficult to control and have preferred $\mathrm{HCl} 0.5 \%$ to $3 \%$ in $70 \%$ ethanol, with or without sodium chloride $0.5 \%$.

The smear is examined dry, though a coverslip placed on the slide avoids "scatter" and aids definition (Matthaei, 1950). For sections a nonfluorescent mounting medium is preferable. Glycerol and ordinary D.P.X. both meet this requirement.

\section{Practical Applications and Results}

The method has been chiefly applied to sputum smears. Tissue sections have had much less attention, being unsuited to control by parallel culture and animal inoculation. Hagemann's original claims were a great shortening of the search for bacilli and many more positive results. Subsequent reports confirmed the saving in time, but the increase in positive results was less spectacular (Besta, 1939; Gärtner, 1939). In a comparison of four techniques-Ziehl-Neelsen, fluorescence, culture, and animal inoculation-Didion (1939) reported most positives from the inoculations. Taking this result as representing $100 \%$ efficiency, the rating of the others was : culture $92 \%$, fluorescence $87.2 \%$, and Ziehl-Neelsen $82.2 \%$. Comparable results were reported by Thomassen 
(1940) and by Schneider (1940). In the same year Finke investigated the auramine staining of various types of acid-fast bacilli. He obtained good fluorescence from all strains of $M$. tuberculosis, none from $M$. smegmatis, occasional fluorescence from $M$. phlei, and faint fluorescence from acid-fast bacilli from milk. These results suggested that acid fastness to carbol fuchsin and auramine respectively might depend on different factors. Richards (1941) stained a sample of mycolic acid-the acid-fast extract of $M$. tuberculosis-with auramine and carbol fuchsin. The auramine was retained much more intensely. It would seem that the link between the bacillus and the two dyes does differ, though, as pointed out by Dubos (1945), factors other than mycolic acid are concerned in the property of acid fastness, especially the physical integrity of the bacterial cell.

Favourable reports on auramine continued (Larsen, 1940 ; Thompson, 1941), though Larsen encountered fallacious results from gastric washings. Kline and Leach (1941) stained dilutions of sputum with carbol fuchsin and auramine. The former was effective up to 1 in 12 , and the latter to 1 in 80 . In the same year reports were made of a minor inconsistency (Lind and Shaughnessy, 1941 ; Oscarsson, 1941 ; Tanner, 1941). These authors found occasional cases positive to carbol fuchsin and negative to auramine. Oscarrson also noted four positive to auramine which were not confirmed by culture or animal inoculation. Richards et al. (1941) and Lind and Shaughnessy (1941) found that with cresol as a preservative for sputum interfering fluorescence was increased, and the former also noted fewer organisms after autoclaving. Richards et al. compared fields in smears stained by auramine and then by carbol fuchsin. Auramine showed more organisms, but carbol fuchsin showed occasional bacilli which had not been shown by auramine.

Dienst (1942) did not support the fluorescence method. He found auramine and carbol fuchsin equally effective in known cases, but he preferred the latter because it showed the cytology, and also because of difficulty in focusing the auramine preparations. Adverse comment was also made by Freiman and Mokotoff (1943), who found an increase in positive results only in gastric contents, a known source of fallacies.

Thompson (1943) investigated a series of cases showing conflicting results with auramine and carbol fuchsin. Of 55 cases, 50 were positive to auramine alone and five to carbol fuchsin alone. In each of these groups $80 \%$ were confirmed by other means, indicating that both stains were equally reliable. In 151 positive smears Van Dyke (1943) reported 41 positive to auramine alone. Of these, all but one were verified by culture.

Ryley (1945) made parallel cultures and inoculations of sputum in cases positive to auramine alone. Not all were confirmed in this way, but he pointed out that, where carbol fuchsin shows very scanty bacilli, culture is sometimes negative. He had no false positives in non-tuberculous cases, and he concluded that a positive result to fluorescence, however scanty the bacilli, should never be ignored. Ritterhoff and Bowman (1945) investigated a similar series of 102 cases. All except four were known to be tuberculous, and were stated to be quiescent, though 68 were classified as advanced or moderate. The specimens included 55 gastric washings and 29 sputa. Only $8.4 \%$ of positives were obtained by culture. The authors state that this is comparable to any series of ZiehlNeelsen-negative specimens, and that the fluorescence results were not consistent with the clinical status of the patients. They question the specificity of the method, and suggest the occurrence of avirulent and non-viable tubercle bacilli, acid-fast saprophytes in the gastric washings, and occasional bacilli in the sputum of inactive cases. Their criticisms would seem, however, to be vitiated by the fact that all but four of their cases were known to be tuberculous.

Another critical report is that of Andrew et al. (1947). They found wide variations in the efficiency of their staff with the auramine method, and also noted eye strain. The illuminant used, however, was the $125 \mathrm{w}$. mercury vapour lamp, which has been shown to be relatively inefficient.

Jennison (1946) introduced a modified ZiehlNeelsen stain, using a green filter in place of the counterstain, and Briggs and Jennison (1948) compared this method with both auramine and ZiehlNeelsen staining. They concluded that the new method was equal to fluorescence, while both were greatly superior to the orthodox Ziehl-Neelsen stain. They recorded that more bacilli were seen by Jennison's method, but with the auramine, permanganate counterstaining was used, which tends to mask the less brilliant organisms. Even so, the carbol fuchsin seemed - to penetrate more effectively. This method has received further support from McCartney (1951), while Jennison and Morgan (1950) concede a degree of superiority to fluorescence. They also note that in concentrating specimens for culture variations in consistency may result in some being under-exposed, with con- 
sequent contamination, and others over-exposed and devitalized. Thus, a negative culture from material positive to fluorescence should not be taken as final.

Further comment on false positives is made by Unholtz (1949), who, in 776 cases, had 23 unconfirmed by culture or inoculation. He refers to the possibility of an increase in false positives from gastric contents after a milk diet, and concludes that, with fluorescence, speed is gained at the expense of accuracy, that unconfirmed results are too many to be explained by devitalized organisms, and that a single positive result is not adequate proof of tuberculosis.

In the case of tissue sections, the unsuitability of auramine alone has already been mentioned (Finke, 1940). This was overcome by counterstaining. Schallock (1940) used thiazol yellow, but the Herrmann method was generally preferred. Tanner (1941) examined 130 paraffin blocks by fluorescence and the Ziehl-Neelsen technique. The auramine gave eight more positives, but, of the Ziehl-Neelsen positives, three were negative to auramine. In a paper on granulomatous prostatitis Tanner and McDonald (1943) used auramine for their positive tuberculous controls. Tanner (1948) compared fields in sections stained with auramine and then by carbol fuchsin. More bacilli were seen by fluorescence, and only rarely did carbol fuchsin show any which had not been shown by auramine. A wide range of tissues showing features typical of or suggestive of tuberculosis was examined by Finke (1950), from which he drew three conclusions, namely (1) bacilli are found more readily in the less typical cases, (2) fluorescence supplements but does not replace ordinary histology, (3) a negative finding does not exclude a diagnosis of tubercle.

\section{Comment}

It is clear that the fluorescence method is not infallible, but this is equally true of others. The chief criticism is that fluorescent organisms which are probably not $M$. tuberculosis are occasionally seen, but even with the Ziehl-Neelsen method acidfast organisms may be found in the sputum of nontuberculous cases (Cory, 1945 ; Medlar, Ordway, and Pesquera, 1943). Possibly some saprophytic or avirulent organisms may fluoresce. Even so, the advantages of the fluorescence method are outstanding, combining ease and speed in observation with simplicity of technique. The investigation of positive results by other methods may always be undertaken.

\section{Present Investigation}

The technique adopted by the writer is that proposed by Matthaei (1950); nevertheless some observations on the apparatus and the procedure may be useful.

The lamp used in the work described later is a 6 v. 18 a. ribbon filament type with a sliding condenser lens. While this gives effective illumination, better results have since been obtained with the $6 \mathrm{v} .5$ a. high intensity lamp supplied by Messrs. Baker for phase-contrast use. Trials have also been made with a 12 v. 2 a. coiled filament high intensity lamp, the $125 \mathrm{w}$. " osira" mercury vapour lamp, a 250 v. $100 \mathrm{w}$. projection lamp, and a 250 v. 500 w. projection lamp. Of these, the first two fail to give full field illumination with low powers (cf. Lempert, 1944), while the $100 \mathrm{w}$. lamp lacks intensity. The $500 \mathrm{w}$. lamp gives a brilliant image, but this is equalled by the Baker lamp referred to above, which is very compact and generates much less heat.

A monocular microscope has been used, with an aplanatic condenser and Leitz objectives of $16 \mathrm{~mm}$., $8 \mathrm{~mm}$. (apochromatic), and $3 \mathrm{~mm}$. dry high power. No interfering fluorescence has been found, and the $8 \mathrm{~mm}$. objective has been adequate for most observations. In practice there is no need for immersion objectives, though they can be used, as fluorescence from the oil is only evident if a drop is viewed with a low power.

The wide range of oculars used by various authors suggests that the power chosen is not important. This, however, has not been my experience, and after trial one of $8 \times$ has been adopted. Lower magnifications, while giving a bright field, give inadequate morphological detail, and higher powers, even $10 \times$, transmit insufficient light. The highest power ocular tested was one of $15 \times$, which was most unsatisfactory. The use of a very high power ocular with a medium power objective was found to be even less desirable for fluorescence than for orthodox microscopy.

Some authors have specified a darkened room, but this is unnecessary, as a light shield of the type illustrated is effective even with bright overhead lights. Only in direct sunlight is any further protection necessary, and a photographer's dark cloth is fully adequate.

The lamp condenser must be adjusted so that the beam is wide enough to give full field illumination with low power objectives, and not, as for dark-ground work, to focus the filament on the microscope mirror. 


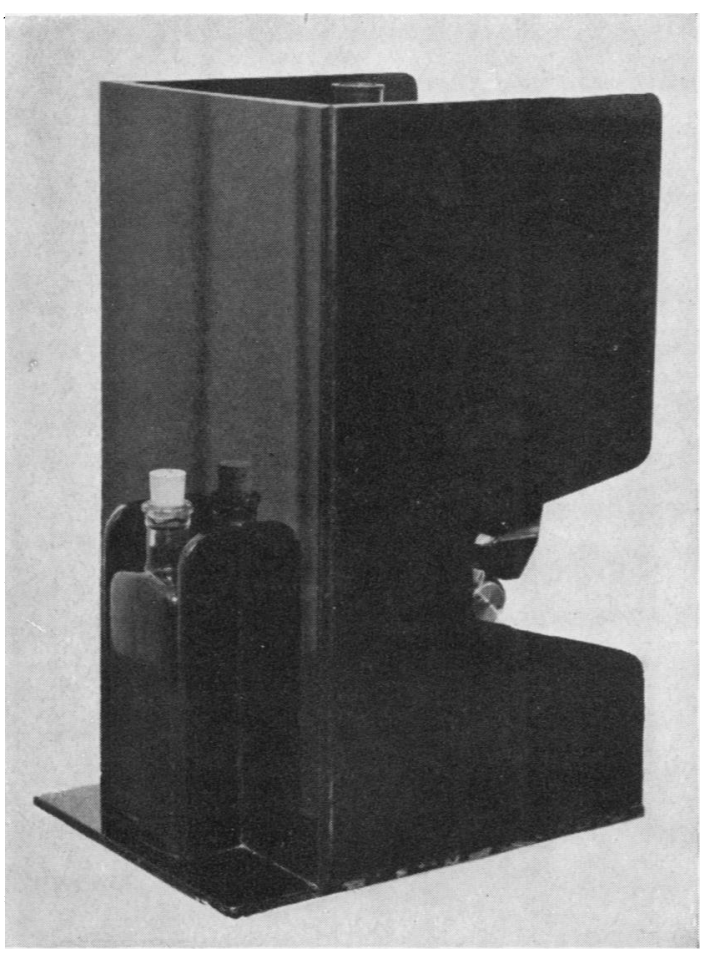

Fig. 2.- Light, shield for microscope constructed from black "perspex" showing cut-away sides for manipulation and blue filter in position.

The blue filter consists of the following solution in an $8 \mathrm{oz}$. medicine bottle.

$\begin{array}{lllll}\begin{array}{l}\text { Hydrated copper sulphate } \\ \text { Concentrated aqueous ammonia }\end{array} & \ldots & 8 \mathrm{~g} . \\ \text { (S.G. 0.88) } & \ldots & \ldots & \ldots & 100 \mathrm{ml} \\ \text { Distilled water } & \ldots & \ldots & \ldots & 160 \mathrm{ml} .\end{array}$

By trial and error it has been found that the optimum concentration is that which gives the brightest image but with no luminous green tinge to the background. Therefore the copper sulphate solution may need diluting.

For the orange filter, a Kodak Wratten gelatin filter type G 15 is cut to shape, and placed in the eyepiece behind the field lens. Care in handling is required, as the gelatin is easily marked. Before centring the lamp and the condenser, the eyepiece is removed, and the tube is racked up a little. The diaphragm remains fully open.

The staining solution is made up as follows:

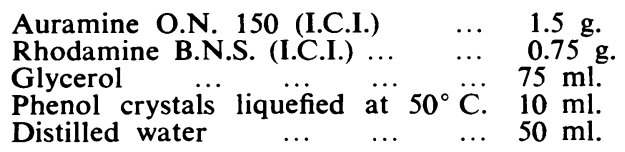

These quantities are half those given by Matthaei, as a large stock is unnecessary for sections, and any risk of deterioration is lessened. Some authors using aqueous auramine have recommended frequent renewal of the stain; Graham (1942) and Hughes (1943) suggest fortnightly renewal, and Lind (1949) even specifies daily preparation, but $I$ have found that the stain given above has been stable over a period of months, even without protection from the light. This is attributed to the high concentration of the dyes, and to the syrupy consistency of the glycerol.

Staining is carried out at $60^{\circ} \mathrm{C}$. for 10 minutes, preferably on a rack over a water bath. After washing in tap water the decolorizing solution $(0.5 \% \mathrm{HCl}$ in $70 \%$ ethanol) is applied with a dropper while the slide is gently rocked. One to two minutes is adequate, then the section is again washed. Staining at room temperature is not effective, and no advantage is to be gained by steaming as for a Ziehl-Neelsen preparation.

No completely effective method of making a permanent preparation has yet been evolved, but, of the methods tried personally, that of evaporating the specimen to dryness and mounting direct in D.P.X. shows negligible fading after eight months. Sections passed through spirit and xylene to the D.P.X. fade in a few weeks, and with watery media in a matter of days. The examination of temporary preparations is usual, however, and glycerol is the medium commonly used.

With the technique described, the bacilli are seen as bright, reddish-gold rods clearly defined against the tissues, which fluoresce green, so giving excellent colour contrast. The structural pattern of the tissue is also obvious. The fluorescence of auramine has been described as golden yellow, yet articles dealing with the combined stain for which improved fluorescence is claimed fail to indicate the precise nature of the improvement. A trial of the original aqueous auramine showed that the bacilli appear as lemon-yellow rods, with no trace of red which would contrast effectively with the tissue background. Any excessive retention of the stain by the tissues can obscure the organisms. The results given by the combined stain are vastly superior, and the contrast between the two methods merits much stronger emphasis than has hitherto been given.

In the past the fluorochrome staining of tissue sections has been undertaken only as a specific research project. Having confirmed the simplicity and effectiveness of the method, the question was considered whether it could play a part in routine histopathology. 
In the histological diagnosis of tubercle there are fundamental contrasts with bacteriological practice. In the latter, while visual demonstration of the bacilli must be supported by culture and animal inoculation, in histology the diagnosis is made from the structural changes in the tissues without reference to the presence of bacilli. The expenditure of time and effort in searching for bacilli in tissue sections might therefore seem of doubtful value, but, as is well known, departures from the classical picture of the tubercle follicle can occur in which the demonstration of bacilli is essential for diagnosis. There are moreover those cases in which features suggestive of tuberculosis are seen in the absence of this infection (Cappell, 1936), and in these, while the negative resu:t for acid-fast bacilli is not conclusive, it does suggest further investigation. Moreover, even in the straightforward cases, seeing the bacilli is a source of satisfaction to both pathologist and clinician. Where the histology is diagnostic it is not essential to delay a report because of additional time spent in searching for bacilli. To summarize, although it is desirable to demonstrate the presence of tubercle bacilli, this is justifiable only if a simple and speedy technique is available.

Preliminary trials with the fluorescence method suggested that this comes close to meeting these requirements. It was decided to investigate all material in the daily surgical routine in which any possibility of tuberculosis arose by the fluorescence method. All material examined was classified as follows, according to the histological appearances: (A) Diagnostic of tuberculosis ; (B) suggestive of tuberculosis, but not conclusive (1, clinically positive, 2, clinically negative); (C) not suggestive of tuberculosis, but suspected clinically ; (D) no appearance or history of tuberculosis : (E) sarcoidosis.

With the surgical material, tissues from a case of miliary tuberculosis were included for control purposes. The fixative used was ordinary corrosive formol. Metcalf and Patton (1944) state that the salts of heavy metals should be avoided, but this finding has not been confirmed in this investigation. The sections were treated with iodine and hypo in the usual way.

Specimens from 120 patients were examined, and the results are shown in Table $I$.

The figures show that even where the histology was diagnostic tubercle bacilli were not commonly seen. Nevertheless an appreciation of the merits of the fluorescence method is best gained by consideration of the features shown by certain groups and individual cases.
TABLE I

ANALYSIS OF RESULTS

\begin{tabular}{c|c|c|c|c|c|c|c}
\hline Groups & All & $A^{*}$ & B1 & B2 & C & D & E \\
\hline $\begin{array}{c}\text { No. specimens } \\
\text {.. }\end{array}$ & 120 & 50 & 13 & 7 & 21 & 16 & 13 \\
\hline No. showing bacilli & 39 & 26 & 6 & - & 2 & - & 5 \\
\hline
\end{tabular}

Group (A): Diagnostic Histology.-As one ڤ would expect, bacilli were seldom found in those $\overrightarrow{0}$ cases showing advanced fibro-caseous and calcified lesions, but even in less chronic cases negative $\vec{\omega}$ results were frequent. Apart from the fatal acute $\frac{\mathscr{\Omega}}{2}$ miliary case, there is no specific histological pic-? ture in which it is probable that bacilli will be found, although there is an increasing probability $\vec{i}$ of doing so when necrobiosis and nuclear karyor- $\omega$ rhexis predominate over endothelial and giant cello formation. To generalize, the greater the departure from the classical tubercle follicle, the $z$ greater the likelihood of finding bacilli. This is in complete accord with the findings of Finke (1950). $\frac{\Phi}{3}$

Certain cases in this group are worthy of individual comment. A biopsy of the tonsil from an adult male showed numerous follic'es in which $\vec{\circ}$ nuclear karyorrhexis was prominent, but whighc were clearly tuberculous on histological groun $\$$. alone. By fluorescence, bacilli were found enormous numbers. This case would normafty have been reported as tuberculous without further® investigation, but the massive nature of the infec- $\stackrel{\mathbb{Q}}{\varrho}$ tion would not have been recognized.

A biopsy of an ulcer of the buccal mucosa from a middle-aged male showed a tuberculous pattern. The site of the lesion was unusual for a tuber culous ulcer, and would have been more consistent with a syphilitic origin, one capable of duplicatingơ the appearances of tuberculosis. Fluorescence ato once showed moderate numbers of acid-fast bacilli Confirmation by culture was obtained and theo Wassermann and Kahn reactions were negative.? In a Ziehl-Neelsen preparation a very few bacillo were found after a protracted search.

Group (A) also included tissues from one patientoㅡ. with tuberculous pericarditis, and from a second patient with miliary tuberculosis. Both received streptomycin treatment and no bacilli were foundo in sections. These results suggest eradication of the infection and more weight can be given to then than to similar findings in Ziehl-Neelsen prepare ations.

Group (B1): Histologically Suggestive, Clinic ally Positive.-In material from 13 patientssix showed acid-fast bacilli. Organisms whicla were scanty in four of these could only have beeक्षे 
found with great difficulty by Ziehl-Neelsen staining. Material from the sixth patient was taken by a bronchial biopsy from a granulomatous area, showing heavy secondary infection, with a layer of fibrinous exudate. Some small, ill-defined follicular structures were present. Fluorescence showed large numbers of acid-fast bacilli, wholly confined to the fibrin layer. It is possible that most of these were saprophytic organisms and confirmation by culture and inoculation would have been desirable. Ziehl-Neelsen staining also showed numerous bacilli, but their location in the fibrin layer was not immediately apparent so that with this method alone the presence of saprophytes might not have been considered. In the (B2) category, with similar histology but no suggestive clinical features, the clinical opinion was supported by the absence of bacilli in all cases.

Group (C) : Histology not Suggestive, Suspected Clinically.-Bacilli were found in material from two patients. In these the histological appearances were those of a pyogenic infection, and tubercle bacilli were scanty.

Group (D): No Histological Appearance or History of Tuberculosis.-These specimens were from a variety of chronic fistulae and sinuses and from other non-specific granulomatous lesions. In no instance were bacilli seen.

Group (E) : Sarcoidosis.-The specimens, all of which were lymph nodes, were taken from filed material, already diagnosed as sarcoidosis. Of 13 cases, five showed acid-fast bacilli. Only one had more than a very occasional bacillus, and in this and in one other case there was early central necrosis in a proportion of the follicles. It is clear that the diagnosis made in these two cases was not justified, but discussion of sarcoidosis is outside the scope of this article. One point does seem clear, however, that the histological demarcation between tuberculosis and sarcoidosis is not sharply defined. If failure to demonstrate acid-fast bacilli is to be taken as a diagnostic criterion, then a considerable number of sections from each case must be examined. The time and effort of doing so by the Ziehl-Neelsen method, together with the likelihood of missing the occasional single bacillus, emphasized the need for a more efficient technique.

The general conclusion to be drawn from this survey is that if acid-fast bacilli are present in a tissue section the fluorescence method will demonstrate them with greater ease and certainty than any other technique at present in use. The apparatus is simple, and does not involve any extensive segregation of equipment. The lamp and the microscope are available at all times for normal use, and only one eyepiece need be permanently reserved. The staining procedure is straightforward, and does not require meticulous timing and supervision, as overstaining is not a problem. The stains are readily obtained and inexpensive.

In the literature on the use of the fluorescence method for sputum, emphasis is laid on the need for additional confirmation, but in sections, if acidfast bacilli are found in tissues showing the histological features of tuberculosis, there need be little reasonable doubt as to their identity. Even in histologically typical sections, however, the finding of bacilli is by no means assured, and for diagnostic purposes it is a waste of time to prolong the examination. Where the histology is typical, the only advantage is that fluorescence shows at a glance whether or not the infection is heavy. Although the likelihood of this is small, just as that of finding no bacilli is considerable, yet the simplicity and speed of the fluorescence method make its routine use justifiable.

No real difficulty has been encountered from artefacts. These do occur, but are generally recognizable as such, and only rarely are they so numerous as to necessitate staining another section. Wollenman, Montgomery, and Foard (1951) described non-tuberculous, acid-fast bacilli in the paraffin used for embedding, but this is hardly to be regarded as a major hazard. Had it been so, it could not have escaped notice for so long.

Of other techniques, a short comparative trial has been made with that propounded by Jennison (1946), to which reference has already been made. This is clearly superior to the orthodox ZiehlNeelsen method, but fluorescence still shows a definite advantage in the speed of recognition of the bacilli. Some of the increased efficiency of Jennison's method is due to the use of the $3.6 \mathrm{~mm}$. objective in place of the $2 \mathrm{~mm}$., but the wider field given still falls far short of that covered by the $8 \mathrm{~mm}$. objective used in the fluorescence method. No obvious increase in the number of bacilli shown by Jennison's method has been noted.

The fluorescence method is of relatively recent origin, and is only now appearing in standard works of reference, but the majority of these deal only with the original auramine stain (Todd and Sanford, 1948 ; Wadsworth, 1947 ; Popper and Szanto, 1950). Of those mentioning rhodamine, Gradwohl (1948) gives the triple stain method of Hughes (1946) without enlarging on that author's comments, but in Recent Advances in Clinical Pathology (Cruickshank, 1951) the auramine- 
rhodamine stain is discussed at some length. Except for the use of lower concentration of these stains than in the work reported here $(50 \%$ approx.), the technique corresponds to that already published. Certain statements must, however, in the writer's opinion, be contested. In particular, the assertion is made that, while the fluorescence method offers great advantages for sputum smears, it is not superior to the ZiehlNeelsen stain for tissue sections. The results in this paper show that this statement is not correct. Another statement in the literature is that the chief advantage of the rhodamine stain lies in the differentiation of artefacts, which are stated not to take on any reddish tinge. The author has not noted this distinction at any time.

A report by Lind (1949) refers to a circular letter sent to 48 laboratories, inviting comment on their experience of the fluorescence method. In reply, 15 laboratories had never used the method, 22 had discarded it, and only two used it as a routine. This seems to constitute a general condemnation of the fluorescence method, but the technique in question was that using auramine alone. One purpose of this paper is to draw attention to the inferiority of this as compared with the auramine-rhodamine combination. Lind emp oyed oculars of $20 \times$ and $30 \times$, another subject of criticism in the present review, and this may have played a part in influencing those who discarded the method. These adverse reports, therefore, should not be taken as having any direct bearing on the technique detailed in these pages.

\section{Conclusions}

In a series of 120 cases of typical or possible tuberculosis, tubercle bacilli have been seen on a number of occasions from the routine use of fluorescence microscopy in circumstances where Ziehl-Neelsen staining would have offered little likelihood of success or where it would not normally have been applied. There is little to be gained by examining typical fibrocaseous lesions, but, if the histological appearances are atypical or there is a secondary pyogenic infection, then fluorescence offers by far the best prospect of demonstrating the presence of acid-fast bacilli. This method may reveal an unsuspectedly heavy infection in cases where early lesions are present. In sarcoidosis an immense saving of time in the examination of numerous sections and much greater certainty in excluding the presence of baciili result from the use of fluorescence, which is also of value in the examination of tissues after antibiotic therapy.

\section{Summary}

The progress and development since 1937 of fluorescence microscopy for $M$. tuberculosis are reviewed. The apparatus is discussed in detail, and the merits of the various components are을 assessed in the light of earlier reports and the $\frac{\bar{\rho}}{\bar{\sigma}}$ writer's experience. Recommendations are made $\stackrel{\mathbb{Q}}{\mathcal{Q}}$ as to the optimum choice of illuminant and optical equipment.

The superiority of the auramine-rhodamine combination has been emphasized, and the results $\vec{\omega}$ obtained from its routine application to a series? of 120 surgical specimens are given. An improvedo method of mounting the sections is described.o

The advantages claimed for the method are that $t_{i}^{+}$ by virtue of its technical simplicity and the ease of $\omega$ observation afforded it can be applied to tissueo sections as a matter of routine. In addition there is no unreasonable increase in the time spent $Z$ in examination. Finally, information can be? obtained by this method which is unobtainable $\stackrel{\mathbb{}}{3}$ by other staining techniques.

I am indebted to Dr. W. B. Davis for his interest $\vec{\oplus}$ and advice in the preparation of this paper. and $\mathscr{C} \omega$ Dr. K. Blum for additional valuable assistance. thanks are also due to Mr. H. C. Gray. A.R.P.S.. dealing with the photography.

\section{REFERENCES}

Andrew, A. B., Wilson, W., and Stuart, R. D. (1947). Glasg med.를 J. 28,220 .

Besta, B. (1939). Ann. Ist. Forlanini., 3, 904

Bogen, E. (1941). Amer. Rev. Tuberc, 44, 267

Briggs, G. O. A., and Jennison, M. H. (1948). Tubercle, 29, 252

Cappell, D. F. (1936). Edinb. med. J., 43, 134.

Cory, R. A. S. (1945). Amer. Rev. Tuberc., 52, 36.

Crossmon, and Loewenstein, E. (1943). J. Lab. clin. Med . 28, $1349 .$.

Cruickshank, D. B. (1951). Recent Advances in Clinical Pathologv 2nd ed., p. 47. Churchill. London

Didion, H. (1939). Klin. Wschr.. 18, 1315

Dienst, R. B. (1942). Amer. J. clin. Path., 12, Tech. Sect. 6. 61. O

Dubos, R. J. (1945). The Bacterial Cell, p. 85. Harvard Universit Press.

Finke, L. (1940). Arch Hyg, Berl . 123, 381

- (1950). Neue med Welt, 16, 577

Freiman, D. G., and Mokotoff, G. F. (1943). Amer. Rev. Tuberc., 48,435 .

Gärtner, H. (1939). Z. Tuberk., 83, 27.

Gradwohl, R. B. H. (1948). Clinical Laboratory Methods and Diagnosis, 4th ed., vol. 2, p. 1529 . Kimpton, London.

Graham, C. F. (1942). J. Lab. clin. Med., 27, 1188.

Graham, C. F. (1942). J. Lab. clin. Med.

Hagemann, P. K. H. (1937). Zbl. Bakt. 1 Abt. Orig., 140, 184

(1938). Münch. med. Wschr. 85, 1066

Herrmann, w. (1938). Dtsch. med. Wschr., 64, 1354

Hughes. G. C. (1943). Med. J. Aust., 2, 353.

(1946). Tubercle, 27, 91 .

Jennison, M. H. (1946). Bull. Inst med. Lab. Tech, 12, 87

.

Kaiserling, C.(1917). Z. Tuberk., 27, 156. Quoted by Ellinger. (1940). Biol. Rev., 15, 323.

Kline, E. K., and Leach, R. E. (1941). J. Bact., 41, 275 Kline, E. K., and Leach, R. E. (1941). J. Bact., 41, 275.
Kloeck, J. M., and Sweany, H. C. (1943). Amer. J. clin. Path, 13 \&

Kolmer, J. A., Spaulding, E. H., and Robinson. H. W. (1951) Approved Laboratory Technic, 5th ed., p. 425. Appleton $=$ Century-Crofts Inc., New York

Larsen, G. (1940). Nord. Med., 8, 1874.

Lee, H. I. (1944). J. Lab. clin. Med., 29, 218.

Lempert, H. (1944). Lancet, 2, 818. 
Lind, H. E. (1949). Amer. J. clin. Path., 19, 72. Matthaei, E. (1950). J. gen. Microbiol. J. Lab. clin. Med., 27, 531.

McCartney, J. E. (1951). Journal of Clinical Pathology, 4, 234.

Medlar, E. M., Ordway, W. H., and Pesquera, G. S. (1943). Amer. Rev. Tuberc., 48, 304.

Metcalf, R. L., and Patton, R. L. (1944). Stain Technol., 19, 11

Norman, W. A., and Jelks, F. W. (1945). Bull. Inst. med. Lab. Tech., $11,37$.

Oscarsson, P. N. (1941). Acta med. scand., 108, 240.

Popper, H., and Szanto, P. B. (1950). In McClung's Handbook of Microscopical Technique, 3rd ed., pp. 678-80. Hoeber, New York.

Richards, O. W. (1941). Science, 93, 190.

Kline, E. K., and Leach, R. E. (1941). Amer. Rev. Tuberc., 44, 255

and Miller, D. K. (1941). Amer. J. clin. Path., 11 Tech. Sect., 5,1 .

Ritterhoff, R. J., and Bowman, M. G. (1945). Ibid., 15, Tech. Sect., 9. 39.
Ryley, J. (1945). Lab. Dig., 9, 1.

Schallock, G. (1940). Münch. med. Wschr., 87, 102

Schneider, P. P. (1940). Z. Tuberk. $84,319$.

Stevens, D. S. (1945). J. Lab. clin. Med., $30,188$.

Tanner, F. H. (1941). Proc. Mayo Clin., 16, 839

- (1948). Amer. J. med. Technol., 14, 83.

- and McDonald, J. R. (1943). Arch. Path., Chicago, 36, 358.

Thomassen, K. (1940). Nord. Med., 8, 1976.

Thompson, L. (1941). Proc. Mayo. Clin., 16, 673.

- (1943). Ibid., 18, 163.

Todd, J. C., and Sanford, A. H. (1948). Clinical Diagnosis by Laboratory Methods, 11 th ed., p. 39. Saunders, Philadelphia.

Unholtz, K. (1949). Z. Tuberk., 93, 305. Van Dyke, A. E. (1943). Amer. J. clin. Path., 13, Tech. Sect.,

Wadsworth, A. B. (1947). Standard Methods, 3rd ed., p. 555. Baillière, Tindall and Cox, London.

Wollenman, O. J., Montgomery, P. O'B., and Foard, T. B. (1951). Amer. J. clin. Path., 21, 295. 